\title{
Properties and Numerical Solutions of Dispersion Curves in General Isotropic Waveguides
}

\author{
Dieter Dobbelaere, Hendrik Rogier, Senior Member, IEEE, Daniël De Zutter, Fellow, IEEE
}

\begin{abstract}
In this paper, some properties of dispersion curves in general isotropic piecewise homogeneous waveguides are rigorously derived. These properties are leveraged in a numerical implementation capable of determining the dispersion curves of such waveguides with cross-section materials that can be highly conductive (such as copper). In a numerical example, the influence of a lossy shielding conductor on the complex modes of a shielded dielectric image guide is investigated for the first time.
\end{abstract}

Index Terms-Dispersion curve, propagation constant, waveguide, shielded dielectric image guide, complex mode

\section{INTRODUCTION}

$\mathbf{T}$ HE recent development of computer-based information systems shows a clear tendency towards higher bit rates and miniaturization of (opto-)electronic components. The design of reliable information-carrying waveguides, driven at high frequencies to sustain the bandwidth requirements, is hampered by the presence of modal dispersion, cross-talk and losses. Accurate prediction of all electromagnetic phenomena occuring in the waveguide cross-section, including the dispersion curves of the eigenmode propagation constants, is of utmost importance for the design and optimization of waveguiding structures. This paper derives properties that contribute to this goal.

There are many techniques available, developed during the past decades, to determine waveguide propagation characteristics. The effective dielectric constant method is an approximate technique that has been used to predict the dispersion relations of several types of dielectric waveguides [1]. However, it only works well under certain assumptions.

The mode-matching technique [2], [3] has the benefits of being both exact (provided a sufficient number of modes is considered) and yielding full modal information, i.e. both propagation constants and modal field distributions. The method requires that the individual constituents of the cross-section are of simple geometrical shape, such that the modes can be determined analytically.

Finite difference and finite element techniques have been succesfully applied to the analysis of dielectric waveguide structures [4], [5]. Advantages of the methods are the easy incorporation of inhomogeneous material regions, where the material parameters can vary continuously as a function of place, and the straightforward determination of the modal characteristics by solving an eigenvalue problem. Although

The authors are with the Electromagnetics Group of the Department of Information Technology of Ghent University and can be contacted at \{dieter.dobbelaere,hendrik.rogier,daniel.dezutter\}@intec.ugent.be its linear system is sparse, the employed volume discretization technique can yield a large number of unknowns in comparison to a boundary element method. Moreover, the analysis of open waveguides is somewhat problematic, as it leads to a larger simulation domain and the introduction of (nonperfect) boundary conditions or an absorbing layer at the boundary. Finally, these methods require a sufficiently fine mesh in regions with rapid field variations, and, therefore, become infeasible in highly conducting regions with skin effect phenomena.

Methods based on the Green's functions of the material regions can be classified into domain or boundary integral methods. The first type was used to study the properties of open planar stratified dielectric waveguides with embedded inhomogeneous material regions [6], [7]. For waveguides with piecewise homogeneous material regions, a boundary integral formulation was applied to analyze dielectric waveguides and lossy multiconductor transmission lines in multilayered media [8], [9]. However, the use of a spectral representation of the Green's function in [8] requires a final spatial inverse Fourier transform, which becomes numerically infeasible for material regions with high losses. For open waveguides, Green's function based methods automatically ensure the radiation conditions at infinity and the prediction of leaky waves. Moreover, less unknowns are needed than with a finite difference or finite element method, as only the inhomogeneous domains and boundaries of piecewise homogeneous domains need to be meshed. Drawbacks of the methods are the non-sparseness of the resulting linear system and the less trivial determination of the dispersion curves, based on the search of the (complex) zeros of the system matrix's determinant.

The study of modal interactions has been thoroughly investigated in the past [10], [11], and the smoothness of the characteric determinant in the dispersion equation is generally taken as an ansatz (based on the physical nature of the problem). In this paper, we investigate the behavior of a single dispersion curve in general isotropic waveguides. The numerical description allows us to explicitly establish the (domain of) holomorphy of the characteristic determinant and prove some useful theorems regarding the properties of dispersion curves. The explicit incorporation of the numerical framework in the derived properties is by no means a limitation, considering existing convergence theorems, but this has not been investigated in this contribution.

A Green's function based boundary element method is employed to calculate the dispersion curves of waveguides with arbitrarily shaped piecewise homogeneous material regions. Use of a technique to evaluate MoM interaction integrals 


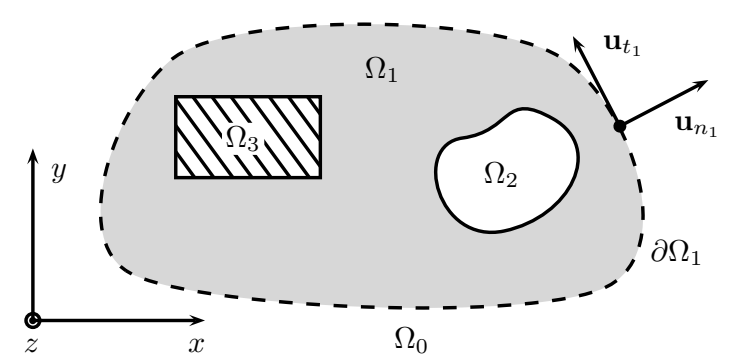

Fig. 1. Waveguide cross section with arbitrarily shaped piecewise homogeneous material regions

in highly conductive materials allows the treatment of both dielectric and electric waveguides with non-perfect conductors. As the formulation is based on a two-dimensional version of the PMCHWT (Poggio-Miller-Chang-Harrington-Wu-Tsai) integral equation [12], [13], [14], spurious modes are nonexistent. It is shown that the evolution of a propagation constant in the complex plane as a function of frequency can be tracked efficiently based on the derived theorems.

In Section II, the geometry of the considered waveguides is presented, along with the employed boundary integral equations. The obtained system of coupled integral equations is then transformed to a finite-dimensional linear system by means of the method of moments (MoM), as explained in Section III. Some properties regarding the MoM system matrix that will be needed in the sequel are also derived. The properties of the dispersion curves that simplify and speed up a numerical implementation are derived in Section IV. Section $\mathrm{V}$ discusses these numerical techniques to search for and track the propagation constants in the complex plane as a function of frequency. Numerical examples demonstrating the correctness and accuracy of the methods are presented in Section VI.

\section{GeOmetry And Integral EQuations}

Consider a waveguide cross section $S \subset \mathbb{R}^{2}$, being the union of linear isotropic piecewise homogeneous material regions $\Omega_{i}$ characterized by their complex electric permittivity $\epsilon_{i}$ and magnetic permeability $\mu_{i}$. We assume that $\mathbf{u}_{n_{i}}$ is the external normal to $\Omega_{i}$ and $\mathbf{u}_{n_{i}} \times \mathbf{u}_{t_{i}}=\mathbf{u}_{z}$ (Fig. 1). Suppose that all the sources and fields have a common time and longitudinal dependence $e^{j(\omega t-\beta z)}(\beta \in \mathbb{C})$, which is omitted for notational convenience. The following representation formulae for the tangential (subscript $t$ ) and longitudinal (subscript $z$ ) components of the electric field at the boundary point $\boldsymbol{\rho}_{b} \in \partial \Omega_{i}$ hold if medium $\Omega_{i}$ contains no sources (with

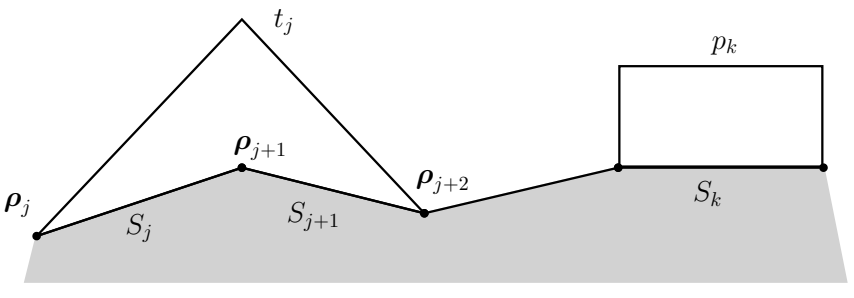

Fig. 2. The boundaries are approximated by means of straight segments, over which triangular and pulse functions are defined

$$
\begin{aligned}
\left.\gamma_{i}^{2}=\omega^{2} \epsilon_{i} \mu_{i}-\beta^{2}\right)[8]: & \\
E_{z}\left(\boldsymbol{\rho}_{b}\right)= & \lim _{\boldsymbol{\rho} \rightarrow \boldsymbol{\rho}_{b}} \oint_{\partial \Omega_{i}} d c^{\prime}\left[E_{z}\left(\boldsymbol{\rho}^{\prime}\right) \frac{\partial G_{i}\left(\boldsymbol{\rho} \mid \boldsymbol{\rho}^{\prime}\right)}{\partial n^{\prime}}\right. \\
& \left.-\left(\frac{j \gamma_{i}^{2}}{\omega \epsilon_{i}} H_{t}\left(\boldsymbol{\rho}^{\prime}\right)-\frac{\beta}{\omega \epsilon_{i}} \frac{\partial H_{z}\left(\boldsymbol{\rho}^{\prime}\right)}{\partial t^{\prime}}\right) G_{i}\left(\boldsymbol{\rho} \mid \boldsymbol{\rho}^{\prime}\right)\right], \\
E_{t}\left(\boldsymbol{\rho}_{b}\right)= & \lim _{\boldsymbol{\rho} \rightarrow \boldsymbol{\rho}_{b}} \oint_{\partial \Omega_{i}} d c^{\prime}\left[\frac{j \omega \mu_{i}}{\gamma_{i}^{2}} H_{z}\left(\boldsymbol{\rho}^{\prime}\right) \frac{\partial^{2} G_{i}\left(\boldsymbol{\rho} \mid \boldsymbol{\rho}^{\prime}\right)}{\partial n \partial n^{\prime}}\right. \\
& +\frac{j \omega \mu_{i}}{\gamma_{i}^{2}}\left(\frac{j \gamma_{i}^{2}}{\omega \mu_{i}} E_{t}\left(\boldsymbol{\rho}^{\prime}\right)-\frac{\beta}{\omega \mu_{i}} \frac{\partial E_{z}\left(\boldsymbol{\rho}^{\prime}\right)}{\partial t^{\prime}}\right) \frac{\partial G_{i}\left(\boldsymbol{\rho} \mid \boldsymbol{\rho}^{\prime}\right)}{\partial n} \\
& -\frac{j \beta}{\gamma_{i}^{2}} E_{z}\left(\boldsymbol{\rho}^{\prime}\right) \frac{\partial^{2} G_{i}\left(\boldsymbol{\rho} \mid \boldsymbol{\rho}^{\prime}\right)}{\partial t \partial n^{\prime}} \\
& \left.+\frac{j \beta}{\gamma_{i}^{2}}\left(\frac{j \gamma_{i}^{2}}{\omega \epsilon_{i}} H_{t}\left(\boldsymbol{\rho}^{\prime}\right)-\frac{\beta}{\omega \epsilon_{i}} \frac{\partial H_{z}\left(\boldsymbol{\rho}^{\prime}\right)}{\partial t^{\prime}}\right) \frac{\partial G_{i}\left(\boldsymbol{\rho} \mid \boldsymbol{\rho}^{\prime}\right)}{\partial t}\right] .
\end{aligned}
$$

The limit should be taken from the inside of $\Omega_{i}$ to the boundary. Similar expressions relating the magnetic field components $H_{z}\left(\boldsymbol{\rho}_{b}\right)$ and $H_{t}\left(\boldsymbol{\rho}_{b}\right)$ at the boundary to the tangential and longitudinal electric and magnetic field components at the boundary are found via the duality substitutions $E \rightarrow H$, $H \rightarrow-E, \epsilon_{i} \rightarrow \mu_{i}$ and $\mu_{i} \rightarrow \epsilon_{i}$ in (1) and (2). The Green's function in the homogeneous medium $i$ is given by $G_{i}\left(\boldsymbol{\rho} \mid \boldsymbol{\rho}^{\prime}\right)=\frac{j}{4} H_{0}^{(2)}\left(\gamma_{i}\left|\boldsymbol{\rho}-\boldsymbol{\rho}^{\prime}\right|\right)$ as the fundamental solution to the two-dimensional Helmholtz operator that satisfies the Sommerfeld radiation condition at infinity, which fixes the choice of the branch cuts of $\gamma_{i}(\beta)$.

The tangential transversal and longitudinal field components $E_{t}, H_{t}, E_{z}$ and $H_{z}$ at the boundaries are the unknowns of the problem. A set of coupled integral equations is obtained by imposing the continuity of these field components at the boundaries between different material regions. If there are sources in $\Omega_{i}$, additional inhomogeneous terms, i.e. not depending on the unknown fields at the boundary, appear in the integral equations that contribute to the right hand side vector of the matrix equation after discretization in the MoM [15]. However, in an eigenmode analysis no sources are present.

\section{Discretized System With The Method of MOMENTS}

\section{A. Properties of the Interaction Integrals}

The boundaries $\partial \Omega_{i}$ are approximated by a union (denoted $\Gamma_{i}$ ) of straight segments $S_{j}$ with length $l_{j}$ and end points $\boldsymbol{\rho}_{j}$ and $\boldsymbol{\rho}_{j+1}$ (Fig. 2), although the use of general curved segments would change nothing to the properties and numerical 


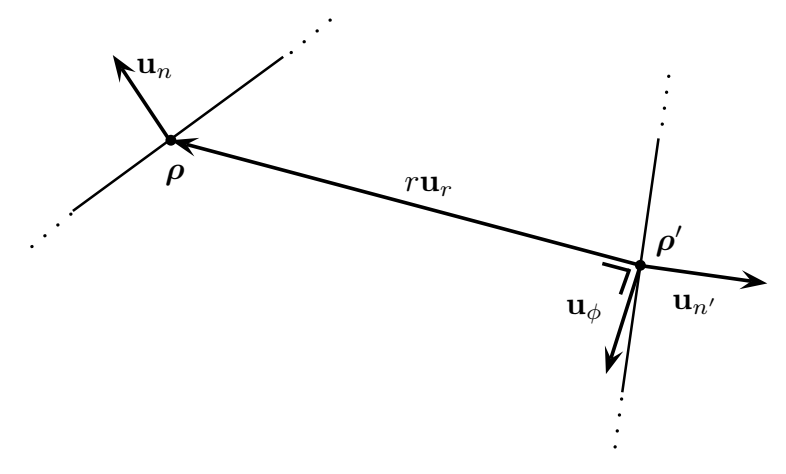

Fig. 3. Relevant to the explicit form of the interaction integrals

techniques derived in the sequel. As in [8], the transverse tangential components $E_{t}$ and $H_{t}$ will be expanded in terms of pulse functions $p_{j}(\boldsymbol{\rho})$, with support over segment $S_{j}$, whereas the longitudinal components $E_{z}$ and $H_{z}$ are expanded into triangular functions $t_{j}(\boldsymbol{\rho})$, with support over $S_{j}$ and $S_{j+1}$ :

$$
\begin{aligned}
& p_{j}(\boldsymbol{\rho})=\frac{1}{l_{j}} \quad \boldsymbol{\rho} \in S_{j}, \\
& t_{j}(\boldsymbol{\rho})= \begin{cases}1-\left|\boldsymbol{\rho}-\boldsymbol{\rho}_{j+1}\right| l_{j}^{-1} & \boldsymbol{\rho} \in S_{j} \\
1-\left|\boldsymbol{\rho}-\boldsymbol{\rho}_{j+1}\right| l_{j+1}^{-1} & \boldsymbol{\rho} \in S_{j+1} .\end{cases}
\end{aligned}
$$

Moreover, a Petrov-Galerkin approach is employed, choosing an identical set of basis and test functions. The integral equations for $E_{z}$ and $H_{z}$ will be tested with pulse functions, whereas the equations for $E_{t}$ and $H_{t}$ will be tested with triangular functions [8]. To calculate the elements in the MoM system matrix, the interaction integrals (5)-(7) below are evaluated numerically for basis and test functions with support over segments that have $\Omega_{i}$ as a neighboring medium. This can easily be seen by inspecting (1) and (2). The tangential derivatives of the Green's function can be transferred to the test function using Stokes' theorem such that only three types of interaction integrals remain.

$$
\begin{aligned}
\mathbf{I}_{j k}^{\mathrm{pp}} & =\int_{\Gamma_{i}} p_{j}(\boldsymbol{\rho}) d c \int_{\Gamma_{i}} G_{i}\left(\boldsymbol{\rho} \mid \boldsymbol{\rho}^{\prime}\right) p_{k}\left(\boldsymbol{\rho}^{\prime}\right) d c^{\prime}, \\
\mathbf{I}_{j k}^{\mathrm{pt}} & =\int_{\Gamma_{i}} p_{j}(\boldsymbol{\rho}) d c \int_{\Gamma_{i}} \frac{\partial G_{i}\left(\boldsymbol{\rho} \mid \boldsymbol{\rho}^{\prime}\right)}{\partial n^{\prime}} t_{k}\left(\boldsymbol{\rho}^{\prime}\right) d c^{\prime}, \\
\mathbf{I}_{j k}^{\mathrm{tt}} & =\int_{\Gamma_{i}} t_{j}(\boldsymbol{\rho}) d c \int_{\Gamma_{i}} \frac{\partial^{2} G_{i}\left(\boldsymbol{\rho} \mid \boldsymbol{\rho}^{\prime}\right)}{\partial n \partial n^{\prime}} t_{k}\left(\boldsymbol{\rho}^{\prime}\right) d c^{\prime} .
\end{aligned}
$$

More explicitly, one can rewrite the interaction integrals as in (8)-(10) (with the notations used in Fig. 3). Note that the inner integral of the last type is to be interpreted in Hadamard finite part sense (p.f.).

$$
\begin{aligned}
\mathbf{I}_{j k}^{\mathrm{pp}}= & \int_{\Gamma_{i}} p_{j}(\boldsymbol{\rho}) \int_{\Gamma_{i}} \frac{j}{4} H_{0}^{(2)}\left(\gamma_{i} r\right) p_{k}\left(\boldsymbol{\rho}^{\prime}\right) d c^{\prime} d c \\
\mathbf{I}_{j k}^{\mathrm{pt}}= & \int_{\Gamma_{i}} p_{j}(\boldsymbol{\rho}) \int_{\Gamma_{i}} \frac{j \gamma_{i}}{4} H_{1}^{(2)}\left(\gamma_{i} r\right)\left(\mathbf{u}_{n^{\prime}} \cdot \mathbf{u}_{r}\right) t_{k}\left(\boldsymbol{\rho}^{\prime}\right) d c^{\prime} d c \\
\mathbf{I}_{j k}^{\mathrm{tt}}= & \int_{\Gamma_{i}} t_{j}(\boldsymbol{\rho}) p \cdot f \cdot \int_{\Gamma_{i}}\left[\frac{j \gamma_{i}^{2}}{8}\left(H_{0}^{(2)}\left(\gamma_{i} r\right)+H_{2}^{(2)}\left(\gamma_{i} r\right)\right) \mathbf{u}_{n} \cdot \mathbf{u}_{n^{\prime}}\right. \\
& \left.\quad-\frac{j \gamma_{i}^{2}}{4} H_{2}^{(2)}\left(\gamma_{i} r\right)\left(\mathbf{u}_{n} \cdot \mathbf{u}_{r}\right)\left(\mathbf{u}_{n^{\prime}} \cdot \mathbf{u}_{r}\right)\right] t_{k}\left(\boldsymbol{\rho}^{\prime}\right) d c^{\prime} d c .
\end{aligned}
$$

Consider a fixed mesh $\Gamma_{i}$ of the boundary $\partial \Omega_{i}$, for all frequencies and propagation constants. The branch cuts $\mathcal{B}_{i}(\omega)$ of the transversal wave number $\gamma_{i}=\sqrt{\omega^{2} \epsilon_{i} \mu_{i}-\beta^{2}}$ at the frequency $\omega$ are chosen on the hyperbola in the complex $\beta$ plane such that $\Im \gamma_{i}<0$ for $\beta \in\left(\mathbb{C} \backslash \mathcal{B}_{i}(\omega)\right)$ [16]. Likewise, denote $\widetilde{\mathcal{B}}_{i}(\beta)$ as the branch cuts of $\gamma_{i}$, for a fixed $\beta$ and viewed as a function of $\omega$, such that $\Im \gamma_{i}<0$ for $\omega \in\left(\mathbb{C} \backslash \widetilde{\mathcal{B}}_{i}(\beta)\right)$. It is easy to see that $\gamma_{i}(\omega, \beta)$ is a holomorphic function of the two variables $\omega$ and $\beta$ in the domain $\mathcal{O}_{i}=\mathbb{C}^{2} \backslash\{(\omega, \beta) \mid \beta \in$ $\left.\mathcal{B}_{i}(\omega)\right\}=\mathbb{C}^{2} \backslash\left\{(\omega, \beta) \mid \omega \in \widetilde{\mathcal{B}}_{i}(\beta)\right\}$. It will be proved in the following theorems that the interaction integrals $(8)-(10)$ are also holomorphic functions of $\omega$ and $\beta$ in $\mathcal{O}_{i}$.

Theorem III.1. Given $\nu \in \mathbb{N}$. Suppose $a(r)$ is a piecewise continuous complex function of a positive real argument $r>0$ that vanishes for $r>R$. Then, if the function $F(\omega, \beta)=$ $\lim _{\epsilon \rightarrow 0} \int_{\epsilon}^{R} a(r) H_{\nu}^{(2)}\left(\gamma_{i}(\omega, \beta) r\right) d r$ converges as an improper Riemann integral in $\mathcal{O}_{i}=\mathbb{C}^{2} \backslash\left\{(\omega, \beta) \mid \beta \in \mathcal{B}_{i}(\omega)\right\}$, it is holomorphic in $\mathcal{O}_{i}$.

Proof. Suppose that $F(\omega, \beta)$ converges as an improper Riemann integral in $\mathcal{O}_{i}$. We will first prove that, for fixed $\omega_{0}$, $F\left(\omega_{0}, \beta\right)$ is holomorphic in the variable $\beta \in \Psi=\mathbb{C} \backslash \mathcal{B}_{i}\left(\omega_{0}\right)$. Consider a fixed $\epsilon>0$. Thanks to the choice of the branch cuts $\mathcal{B}_{i}\left(\omega_{0}\right), H_{\nu}^{(2)}\left(\gamma_{i}\left(\omega_{0}, \beta\right) r\right)$ is holomorphic for $r>0$ and $\beta \in$ $\Psi$. As by assumption $F_{\epsilon}(\beta)=\int_{\epsilon}^{R} a(r) H_{\nu}^{(2)}\left(\gamma_{i}\left(\omega_{0}, \beta\right) r\right) d r$ is Riemann integrable in $\Psi$, the sequence of its holomorphic Riemann sums converges uniformly on all compact subsets of $\Psi$. Therefore, for each value of $\epsilon$, the limit of this sequence of holomorphic Riemann sums, namely $F_{\epsilon}(\beta)$, is found to be holomorphic too, by a well-known theorem of Weierstrass. As $\lim _{\epsilon \rightarrow 0} F_{\epsilon}(\beta)$ exists, the sequence of the holomorphic functions $F_{1 / n}(\beta)$ for $n \rightarrow \infty$ also converges uniformly on all compact subsets of $\Psi$, implying that the limit function $F\left(\omega_{0}, \beta\right)=\lim _{\epsilon \rightarrow 0} F_{\epsilon}(\beta)$ is holomorphic in $\Psi$.

Completely analogously, one can prove that, for fixed $\beta_{0}$, $F\left(\omega, \beta_{0}\right)$ is holomorphic as a function of $\omega$ in $\mathbb{C} \backslash \widetilde{\mathcal{B}}_{i}\left(\beta_{0}\right)$. Application of Hartogs' fundamental theorem, also called the separate analyticity theorem [17], leads to the proposition.

Theorem III.2 (Holomorphy of the interaction integrals). The interaction integrals (8) - (10) are holomorphic functions of the two complex variables $\omega$ and $\beta$ in the domain $\mathcal{O}_{i}$.

Proof. It can be shown that interactions of the form (8) and (9) can be reduced to the form of $F(\omega, \beta)$ in Theorem III.1, which converges as an improper Riemann integral in 
$\mathcal{O}_{i}$. Interactions of the last form (10) cannot be written straightforwardly as an improper Riemann integral if the kernel in the integration domain contains a hypersingularity. By extracting the hypersingular part of the second order Hankel function, the regularised integrand is (improperly, if basis and test functions are overlapping or tangent) Riemann integrable, and by arguments similar to the proof in Theorem III.1, the integral leads to a holomorphic function in $\mathcal{O}_{i}$. The finite part integral of the hypersingular part is independent of $\omega$ and $\beta$, and thus trivially holomorphic. Indeed, it is given by:

$$
\begin{aligned}
\left.\right|_{j k, \text { sing }} ^{\mathrm{tt}}= & -\int_{\Gamma_{i}} t_{j}(\boldsymbol{\rho}) d c \quad p \cdot f \cdot \int_{\Gamma_{i}} \frac{1}{2 \pi r^{2}}\left[\mathbf{u}_{n} \cdot \mathbf{u}_{n^{\prime}}\right. \\
& \left.-2\left(\mathbf{u}_{n} \cdot \mathbf{u}_{r}\right)\left(\mathbf{u}_{n^{\prime}} \cdot \mathbf{u}_{r}\right)\right] t_{k}\left(\boldsymbol{\rho}^{\prime}\right) d c^{\prime} .
\end{aligned}
$$

This lets us conclude that the interaction integrals are holomorphic functions of $\omega$ and $\beta$ in $\mathcal{O}_{i}$.

\section{B. Properties of the System Matrix}

We denote the MoM system matrix at frequency $\omega$ and longitudinal wave number $\beta$ as $\mathrm{M}(\omega, \beta) \in \mathbb{C}^{N \times N}$, i.e. it has the same dimension $N$ for all $\omega$ and $\beta$, resulting from the earlier assumed fixed discretization of the boundaries $\partial \Omega_{i}$. Its determinant will be designated as $\mathcal{D}(\omega, \beta)=\operatorname{det} \mathrm{M}(\omega, \beta)$. The employed methods to search for propagation constants of the waveguide, discussed in Section IV, rely on the determination of the zeros of $\mathcal{D}(\omega, \beta)$ in the $\beta$-plane. A few interesting properties of the dispersion curves as a function of frequency can be proved, taking into account the holomorphy of the determinant. The latter is the subject of the following theorems.

Theorem III.3 (Holomorphy of the matrix elements). The matrix elements of $\mathrm{M}(\omega, \beta)$ are holomorphic functions of $\omega$ and $\beta$ in the domain $\mathcal{O}=\left(\mathbb{C}_{0} \times \mathbb{C}\right) \backslash\left(\cup_{i}\left\{(\omega, \beta) \mid \beta \in \mathcal{B}_{i}(\omega)\right\}\right)$.

Proof. The discretized linear system arises after expanding the tangential boundary field components into a set of basis functions and imposing moments of the continuity relations, arising from the representation formulae (1)-(2) and their duals, as explained in Section II. Therefore each matrix element can be written as a finite sum of products of prototype integrals (holomorphic in $\cap_{i} \mathcal{O}_{i}$, see Theorem III.2) with functions that are holomorphic in $\mathcal{O}$ (note that some factors have a pole at $\omega=0$ ). This completes the proof.

Theorem III.4 (Holomorphy of the determinant). The system matrix determinant $\mathcal{D}(\omega, \beta)$ is holomorphic as a function of $\omega$ and $\beta$ in the domain $\mathcal{O}$, defined in Theorem III.3.

Proof. This follows immediately from Theorem III.3 and the fact that the determinant is a finite sum of finite products of matrix elements (up to a sign).

The following theorem discusses the symmetry of the matrix determinant as a function of $\beta$, if the cross-section consists entirely of lossless materials. This property implies that the numerically found complex waves always come in pairs with complex conjugate propagation constants.
Theorem III.5 (Symmetry property of the determinant for lossless structures). Suppose $\epsilon_{i}, \mu_{i} \in \mathbb{R}_{0}$ and $(\omega, \beta) \in \mathcal{O}$, then $\mathcal{D}(\omega, \bar{\beta})=\overline{\mathcal{D}(\omega, \beta)}$, where $\bar{a}$ stands for the complex conjugate of $a$.

Proof. We shall make use of the identity $H_{n}^{(2)}(-\bar{z})=$ $(-1)^{n+1} \overline{H_{n}^{(2)}(z)}$ if $\Im z<0$ and $n \in \mathbb{Z}$, which can be proved using elementary properties of Bessel functions [18]. When we replace $\beta$ with $\bar{\beta}$ in the interaction integrals (8)-(10), we can use the previous identity for $(\omega, \beta) \in \mathcal{O}$, as then $\Im\left(\gamma_{i} r\right)<0$. Note that in the process of replacing $\beta \rightarrow \bar{\beta}$, we have $\gamma_{i} \rightarrow-\overline{\gamma_{i}}$ and $\gamma_{i}^{2} \rightarrow \overline{\gamma_{i}^{2}}$. This lets us conclude that $\mathrm{I}_{j k}^{\mathrm{pp}} \rightarrow \overline{\mathrm{I}_{j k}^{\mathrm{pp}}}, \mathrm{I}_{j k}^{\mathrm{pt}} \rightarrow \overline{\mathrm{I}_{j k}^{\mathrm{pt}}}$ and $\mathrm{I}_{j k}^{\mathrm{tt}} \rightarrow \overline{\mathrm{I}_{j k}^{\mathrm{tt}}}$ if $\beta \rightarrow \bar{\beta}$. By examining the additional factors in the representation formulae (1)-(2) and their duals, it can be seen that some contributions, for example $H_{t}$ as a source for $E_{z}$ (denoted $\left[E_{z} H_{t}\right]$ ), will be transformed according to $\left[E_{z} H_{t}\right] \rightarrow-\overline{\left[E_{z} H_{t}\right]}$. Others, for example $E_{z}$ as a source for $E_{z}$, will be transformed according to $\left[E_{z} E_{z}\right] \rightarrow \overline{\left[E_{z} E_{z}\right]}$. However, $\mathrm{M}(\omega, \bar{\beta})$ and $\overline{\mathrm{M}(\omega, \beta)}$ will only differ up to a multiplication of the same number of columns and rows with -1 . This leads to the proposition.

\section{Properties of the Dispersion Curves}

Given $\left(\omega_{0}, \beta_{0}\right) \in \mathcal{O}$, with $\omega_{0} \in \mathbb{R}_{0}^{+}$. Suppose that $\beta_{0}$ is a zero of the determinant at the operating frequency $\omega_{0}$, i.e. $\mathcal{D}\left(\omega_{0}, \beta_{0}\right)=0$. In the current context, $\beta_{0}$ corresponds to the propagation constant of one or more eigenmodes that can exist in the structure. As a consequence of Hartogs' lemma, also called the Osgood-Brown theorem [17], the zero $\left(\omega_{0}, \beta_{0}\right)$ cannot be an isolated one. The Weierstrass preparation theorem and the fact that $\mathcal{D}(\omega, \beta)$ may be expressed a power series around $\left(\omega_{0}, \beta_{0}\right)$, which has a term only involving $\beta-\beta_{0}$, independent of $\omega-\omega_{0}$ (otherwise $\mathcal{D}\left(\omega_{0}, \beta\right)$ would be identically zero in the neighborhood where the power series holds, and thus, by analytic continuation, the determinant would vanish for $\omega=\omega_{0}$ ), allows to write the following local representation for $\mathcal{D}(\omega, \beta)$, valid in a neighborhood ${ }^{1}$ $U_{\epsilon}=B\left(\omega_{0}, \epsilon\right) \times B\left(\beta_{0}, \epsilon\right)$ of $\left(\omega_{0}, \beta_{0}\right)[19]$ :

$$
\mathcal{D}(\omega, \beta)=\left[\left(\beta-\beta_{0}\right)^{\nu}+\sum_{i=0}^{\nu-1} c_{i}(\omega)\left(\beta-\beta_{0}\right)^{i}\right] \phi(\omega, \beta),
$$

where $\phi(\omega, \beta)$ is holomorphic and zero-free in $U_{\epsilon}, \nu \in \mathbb{N}_{0}$ and the functions $c_{i}(\omega)$ are holomorphic in a neighborhood of $\omega_{0}$ and vanish at $\omega_{0}$. From (11), it is clear that the zero $\beta_{0}$ of the function $\mathcal{D}\left(\omega_{0}, \beta\right)$ has multiplicity $\nu$. The following theorem describes the behavior of this zero as a function of $\omega$, in a neighborhood of $\omega_{0}$ on the real axis.

Theorem IV.1 (Behavior of a dispersion curve as a function of frequency). Suppose that $\left(\omega_{0}, \beta_{0}\right)$ is a zero of $\mathcal{D}(\omega, \beta)$ of multiplicity $\nu$, with $\omega_{0} \in \mathbb{R}_{0}^{+}$and $U_{\epsilon}$ a neighborhood of $\left(\omega_{0}, \beta_{0}\right)$ where (11) holds. If, for each $\omega$ in a real interval $(a, b) \subset\left(\omega_{0}-\epsilon, \omega_{0}+\epsilon\right)$ (with $\left.b>a\right)$, it holds that $\mathcal{D}(\omega, \beta)$ has exactly one zero $\left(\omega, \beta_{1}(\omega)\right)$ with multiplicity $\nu$ in $U_{\epsilon}$, then the same holds for all $\omega \in B\left(\omega_{0}, \epsilon\right)$, for which $\mathcal{D}(\omega, \beta)$ has

\footnotetext{
${ }^{1} B\left(z_{0}, \epsilon\right)=\left\{z \in \mathbb{C}:\left|z-z_{0}\right|<\epsilon\right\}$, is the open disk centered at $z_{0}$ with radius $\epsilon$, whereas $\bar{B}\left(z_{0}, \epsilon\right)$ denotes its closure.
} 
zeros inside $U_{\epsilon}$. Moreover, the dispersion curve of the zero $\beta_{1}(\omega)$ inside $U_{\epsilon}$ will be smooth.

Proof. For $\omega \in(a, b)$, there is only one zero with multiplicity $\nu$ inside $U_{\epsilon}$. This means that the term in brackets in (11) is uniquely factorized in the following way, for $\omega \in(a, b)$ :

$$
\begin{gathered}
\left(\beta-\beta_{0}\right)^{\nu}+\sum_{i=0}^{\nu-1} c_{i}(\omega)\left(\beta-\beta_{0}\right)^{i}=\left(\beta-\beta_{1}(\omega)\right)^{\nu} \\
=\sum_{i=0}^{\nu}\left(\begin{array}{c}
\nu \\
i
\end{array}\right)(-1)^{\nu-i}\left(\beta-\beta_{0}\right)^{i}\left(\beta_{1}(\omega)-\beta_{0}\right)^{\nu-i} .
\end{gathered}
$$

This implies that $c_{i}(\omega)=\left(\begin{array}{c}\nu \\ i\end{array}\right)(-1)^{\nu-i}\left(\beta_{1}(\omega)-\beta_{0}\right)^{\nu-i}$ for $i=0,1, \ldots, \nu-1$. Or, equivalently, the following relations hold:

$$
\begin{array}{r}
\beta_{1}(\omega)=\beta_{0}-\frac{1}{\nu} c_{\nu-1}(\omega), \\
c_{i}(\omega)=\left(\begin{array}{c}
\nu \\
i
\end{array}\right)\left(\frac{c_{\nu-1}(\omega)}{\nu}\right)^{\nu-i} .
\end{array}
$$

As the interval $(a, b)$ contains a limit point, the relations (15) will hold for all $\omega \in B\left(\omega_{0}, \epsilon\right)$. Thus, the factorisation (12) holds in the whole neighborhood $U_{\epsilon}$, implying that for all $\omega \in B\left(\omega_{0}, \epsilon\right)$, for which $\mathcal{D}(\omega, \beta)$ has zeros inside $U_{\epsilon}$, the zero is unique and has multiplity $\nu$. From (14), it clear that $\beta_{1}(\omega)$ is holomorphic in $B\left(\omega_{0}, \epsilon\right)$ and thus smooth for $\omega \epsilon$ $\left(\omega_{0}-\epsilon, \omega_{0}+\epsilon\right)$.

Corollary IV.2 (Dispersion curves cannot split up by themselves). Suppose that $\beta_{1}(\omega)$ is a complex-valued function of real argument $\omega \in\left(\omega_{0}-\epsilon, \omega_{0}\right]$, representing a zero of the determinant with constant multiplicity $\nu$, i.e. $\mathcal{D}\left(\omega, \beta_{1}(\omega)\right)=0$. Then there exists a $\delta>0$ such that $\beta_{1}(\omega)$ can be smoothly extended to the interval $\omega \in\left(\omega_{0}-\epsilon, \omega_{0}+\delta\right)$, still obeying $\mathcal{D}\left(\omega, \beta_{1}(\omega)\right)=0$ with multiplicity $\nu$. Therefore, dispersion curves cannot split up by themselves. There has to be at least an intersection with another curve or an intersection with a branch cut $\mathcal{B}_{i}(\omega)$.

\section{NumericAl TeChNiques}

The location of the propagation constants in the complex plane, as found with the argument principle, is refined using an adaptive quadrature scheme with adjustable accuracy. The tracking of a propagation constant as a function of frequency is eased by considering the derived theorems.

\section{A. Argument Principle}

As a first coarse estimate of the distribution of the propagation constants for a fixed angular frequency $\omega$, we use Cauchy's argument principle (as in [20]). The regions where propagation constants are likely to be found are meshed into rectangular boxes that contain no branch cuts. The argument principle can then be used to determine the number of zeros of the determinant function, counted with their multiplicity, inside a rectangular box $R$ in the $\beta$-plane with the sole knowledge of the function values on the boundary. Indeed, if $R \subset\left(\mathbb{C} \backslash \cup_{i} \mathcal{B}_{i}(\omega)\right.$ ) (no branch cuts through $R$ ) and $\mathcal{D}(\omega, \beta) \neq 0$ for $\beta \in \partial R$ (no zero on the boundary), then the determinant function has the following number of zeros inside $R$, where the contour integral is taken in counterclockwise direction:

$$
n_{R}=\frac{1}{2 \pi j} \oint_{\partial R^{+}} \frac{\partial_{\beta} \mathcal{D}(\omega, \beta)}{\mathcal{D}(\omega, \beta)} d \beta .
$$

The winding number $n_{R}$ of the determinant function along $\partial R$ and is equal to the number of times the curve $\{\mathcal{D}(\omega, \beta) \mid \beta \in$ $\partial R\}$ circles around the origin. This last property can be easily calculated numerically by inspecting the change in argument of the determinant function between neighboring sample points on the boundary [21].

\section{B. Adaptive Quadrature Scheme}

A more accurate estimate of the distribution of the propagation constants can be obtained by subsequently refining boxes with $n_{R}>0$ and applying the argument principle. However, this approach becomes infeasible if one wants to know the location of the zeros up to a fixed (and high) accuracy. To solve this issue, the coarse search with the argument principle is followed by a refinement step, consisting of numerically evaluating the following integral:

$$
\bar{z}=\frac{1}{2 \pi n_{R} j} \oint_{\partial R^{+}} \beta \frac{\partial_{\beta} \mathcal{D}(\omega, \beta)}{\mathcal{D}(\omega, \beta)} d \beta .
$$

If there is only one zero with multiplicity $n_{R}$ inside $R$, then $\bar{z}$ precisely represents the location of this zero. Otherwise, $\bar{z}$ represents the centre of mass of the enclosed zeros, weighted with their multiplicities. A sufficient refinement of the boxes via the argument principle is able to exclude the latter case. We have implemented a numerical quadrature scheme, capable of evaluating (17) to a given precision of the real and imaginary part, taking into account the rather high cost of evaluating the determinant function in a point. These considerations lead to a Gauss-Kronrod quadrature rule, as the addition of $N+1$ Kronrod nodes to the $N$ Gauss-Legendre nodes in order to estimate the error of the original Gauss-Legendre rule, allows to reuse the function values at the Gauss-Legendre nodes.

In this way, the integral in (17) is split up into four parts, with each one corresponding to a segment of $\partial R$. The integrand of such a segment integral is smooth (Theorem III.4) and the derivative $\partial_{\beta} \mathcal{D}(\omega, \beta)$ at the $2 N+1$ quadrature points is numerically estimated by a linear transformation of the determinant values at the nodes, which is essentially a projector of the function values at the quadrature nodes onto a set of orthogonal polynomials, followed by a derivative operator of the polynomials at the same nodes. If the error of the segment integral is too high to guarantee the accuracy of the end result, the segment is divided into two equal segments where the same procedure is performed [22]. The quadrature scheme can thus be classified as an adaptive Gauss-Kronrod scheme.

\section{Frequency sweep}

The numerical procedure of tracking the dispersion curve of a propagation constant as a function of frequency is greatly facilitated by the use of previously derived theorems. At the start of the frequency sweep, we choose a fixed mesh along 
the material boundaries that is fine enough to accurately represent the boundary and EM wave behavior at the considered frequencies. In this way, the curve $\beta(\omega)$ we are looking for, starting from a seed value $\beta_{0}$ at $\omega_{0}$, will be smooth as a function of frequency (Theorem IV.1). This allows the use of an extrapolation method to accurately estimate the location of the propagation constant at the next frequency point. This prediction will then be refined by using the adaptive quadrature scheme, discussed in the previous paragraph.

The property that the winding number of the curve $\beta(\omega)$ stays constant, as a consequence of Theorem IV.1 (unless two curves cross or a branch cut is met), is used to check if the box around the estimation contains no extra propagation constants. If the box around the estimated propagation constant at the next frequency point is chosen too large, such that another zero of the determinant lies inside it, formula (17) will give the centre of mass of the enclosed zeros, and not the sought value. However, this case can be detected as the winding number increases, which signals the numerical procedure to reduce the box.

\section{EXAMPLES}

\section{A. Complex Modes in a Shielded Dielectric Image Guide}

In this example, the presented boundary element method is employed to numerically determine the dispersion curves of complex modes. These modes can arise in certain inhomogeneous waveguides, and have propagation constants with nonzero real and imaginary part, even if all material regions in the cross section are lossless. Consider the shielded dielectric image guide of Figure 4, which is essentially a rectangular waveguide loaded with a dielectric material region with relative permittivity $\epsilon_{r}=9$, located at the bottom conductor.

It is well-known that complex modes can exist in this type of waveguide [5]. If the shielding conductor $\Omega_{c}$ is a perfect electric conductor (PEC), there are no losses inside the structure, which is reflected by the fact that complex modes exist in pairs with conjugate propagation constants [23], [24]. In the lossless case, the complex conjugate symmetry of the propagation constants of the mode pair is nicely predicted by the numerical framework (see Theorem III.5). If the dielectric load material is lossy, the propagation constants are no longer each other's complex conjugate [5]. We will show that the same applies for the case with non-perfectly conducting waveguide walls and lossless dielectric load material.

To illustrate the applicability of the boundary element method to a highly conductive region, consider a non-perfect shielding conductor $\Omega_{c}$ with electric conductivity $\sigma$ and thickness $t=$ $1 \mathrm{~mm}$. In the numerical examples, we study the effect of the conductivity by choosing two distinct values $\sigma_{a}=5.8 \mathrm{e} 7 \mathrm{~S} / \mathrm{m}$ (copper) and $\sigma_{b}=10^{-4} \sigma_{a}$. We use the same geometry as in [5], i.e. $a=2 b=15.798 \mathrm{~mm}, w=6.9 \mathrm{~mm}$ and $h=3.2 \mathrm{~mm}$. Figures 5 and 6 show the normalized real and absolute value of the normalized imaginary part of two propagation constant curves, termed $\beta_{1}(f)$ and $\beta_{2}(f)$, for the two conductivities, with the frequency $f=\omega / 2 \pi$ varying between $12 \mathrm{GHz}$ and $15 \mathrm{GHz}$.

In the waveguide with PEC boundary, a true bifurcation of

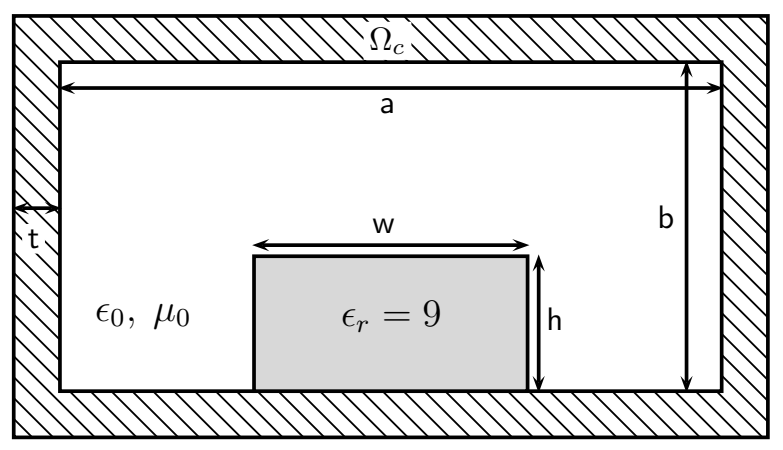

Fig. 4. Cross section of a shielded dielectric image guide

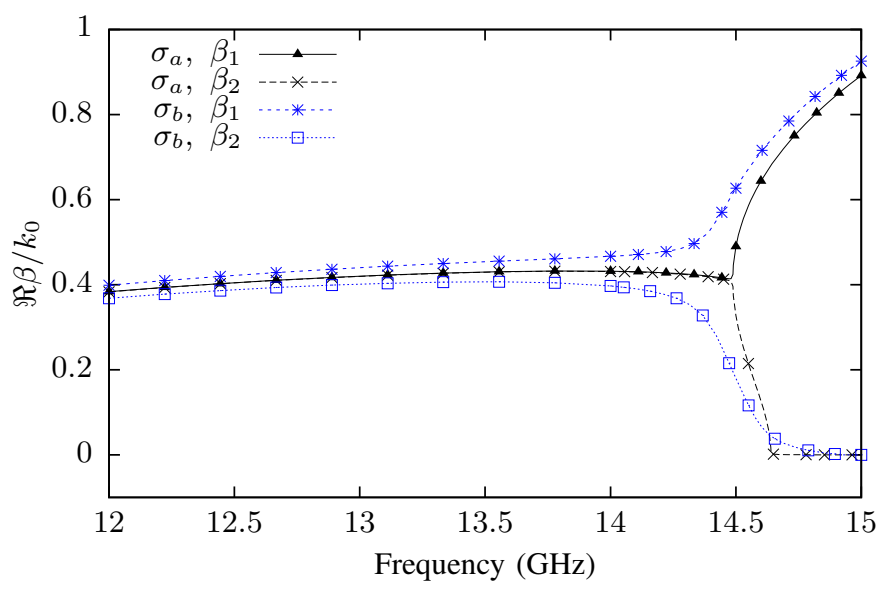

Fig. 5. Real part of the normalized dispersion curves for different conductivities

the complex modes occurs around $f_{b}=14.5 \mathrm{GHz}$, and the modes are exactly each other's complex conjugate before the bifurcation, i.e. $\beta_{1}(f)=\overline{\beta_{2}(f)}$ if $f<f_{b}$ [5]. At first glance, the bifurcation for a lossless waveguide with PEC boundary seems to be in contradiction with Corollary IV.2. However, this bifurcation happens on the branch cuts of the transversal wave numbers of both lossless material regions (free space and the dielectric load material), i.e. on the real axis in the complex $\beta$-plane around $0.4 k_{0}$, and Theorem IV.1 only holds for $\left(\omega_{0}, \beta_{0}\right) \in \mathcal{O}$, i.e. outside the branch cuts $\mathcal{B}_{i}(\omega)$.

For the considered case of a lossy shielding conductor, the bifurcation disappears and the two modes are no longer conjugate (Fig. 5 and 6). It was verified that by increasing the conductivity of the shielding conductor, the dispersion curve $\beta_{1}(f)$ converges to $\overline{\beta_{2}(f)}$ for $f<f_{b}$. This can be expected, as a PEC boundary corresponds to the case $\sigma \rightarrow \infty$. As the curves $\beta_{1}(f)$ and $\beta_{2}(f)$ do not cross a branch cut, and because they cannot cross each other (both lie on the opposite side of the branch cuts), no bifurcation is allowed (Corollary IV.2). Moreover, their curves in the complex plane are smooth, but this is not really apparent from Figures 5 and 6. However, Figures 7 and 8 clearly demonstrate this fact, by showing the logarithm of the real part and the absolute value of the normalized imaginary part of both curves. 


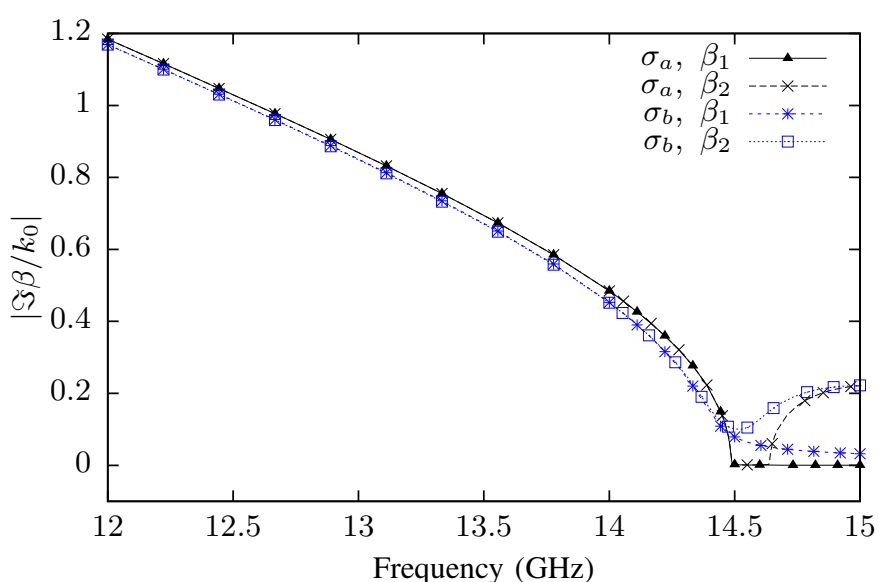

Fig. 6. Absolute value of the imaginary part of the normalized dispersion curves for different conductivities

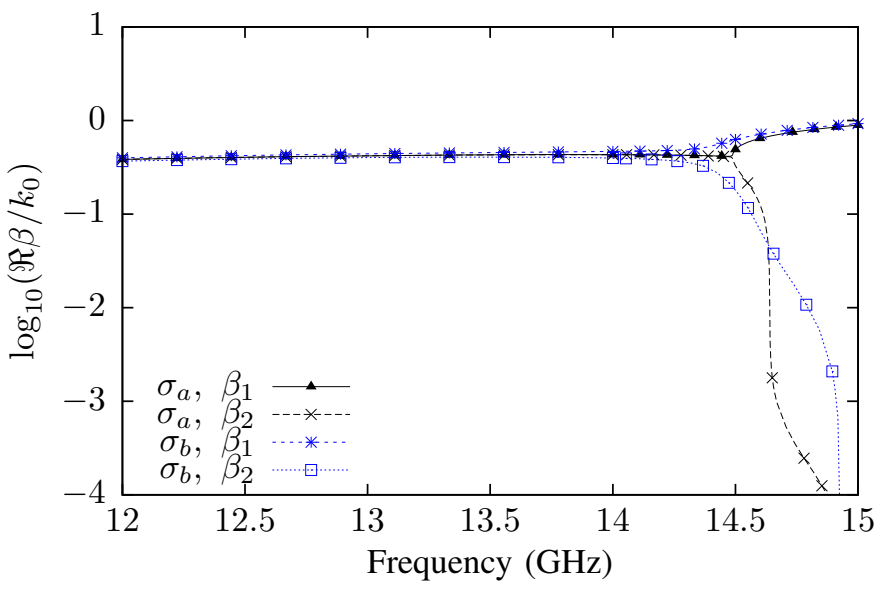

Fig. 7. Logarithm of the real part of the normalized dispersion curves for different conductivities

\section{CONCLUSION}

Theorems were presented stating some properties of the dispersion curves for general isotropic piecewise homogeneous waveguides. These theorems were leveraged in a numerical implementation of a set of boundary integral equations to determine the waveguide eigenmodes and their propagation constants. These boundary integral equations can handle highly conductive materials (such as copper).

A challenging numerical example demonstrates the possibilities of our tool. To the authors' knowledge, this is the first time that the effect of a lossy shielding conductor on the complex modes of a shielded dielectric image guide has been numerically investigated.

\section{REFERENCES}

[1] W. McLevige, T. Itoh, and R. Mittra, "New waveguide structures for millimeter-wave and optical integrated circuits," Microwave Theory and Techniques, IEEE Transactions on, vol. 23, no. 10, pp. 788 - 794, Oct. 1975.

[2] R. Mittra, Y.-L. Hou, and V. Jamnejad, "Analysis of open dielectric waveguides using mode-matching technique and variational methods," Microwave Theory and Techniques, IEEE Transactions on, vol. 28, no. 1, pp. 36 - 43, Jan. 1980

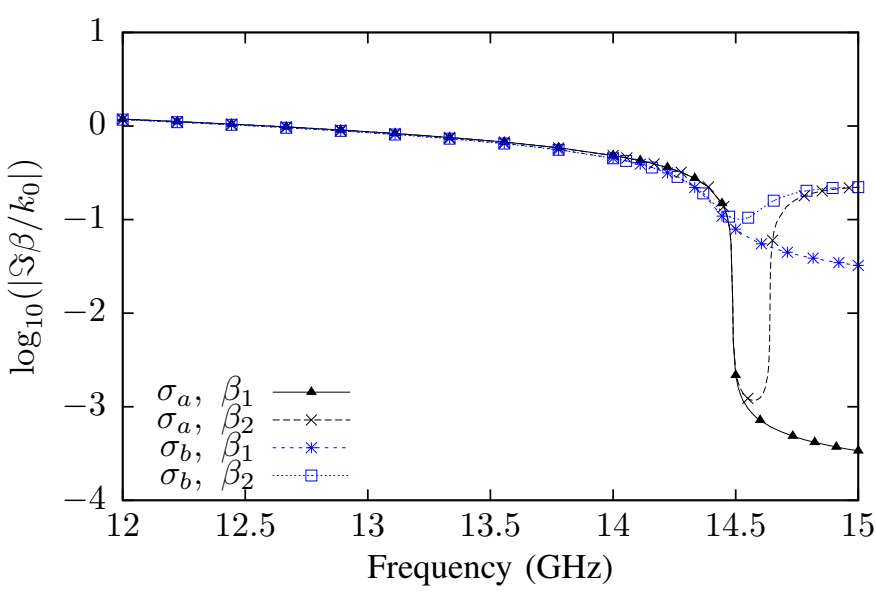

Fig. 8. Logarithm of absolute value of the imaginary part of the normalized dispersion curves for different conductivities

[3] J. Strube and F. Arndt, "Rigorous hybrid-mode analysis of the transition from rectangular waveguide to shielded dielectric image guide," Microwave Theory and Techniques, IEEE Transactions on, vol. 33, no. 5, pp. 391 - 401, May 1985.

[4] K. Bierwirth, N. Schulz, and F. Arndt, "Finite-difference analysis of rectangular dielectric waveguide structures," Microwave Theory and Techniques, IEEE Transactions on, vol. 34, no. 11, pp. 1104 - 1114, Nov. 1986.

[5] F. Fernandez, Y. Lu, J. Davies, and S. Zhu, "Finite element analysis of complex modes in inhomogeneous waveguides," Magnetics, IEEE Transactions on, vol. 29, no. 2, pp. 1601 -1604, Mar. 1993.

[6] J. Bagby, D. Nyquist, and B. Drachman, "Integral formulation for analysis of integrated dielectric waveguides," Microwave Theory and Techniques, IEEE Transactions on, vol. 33, no. 10, pp. 906 - 915, Oct. 1985.

[7] J.-F. Kiang, S. Ali, and J. Kong, "Integral equation solution to the guidance and leakage properties of coupled dielectric strip waveguides," Microwave Theory and Techniques, IEEE Transactions on, vol. 38, no. 2 , pp. $193-203$, Feb. 1990.

[8] F. Olyslager, D. De Zutter, and K. Blomme, "Rigorous analysis of the propagation characteristics of general lossless and lossy multiconductor transmission lines in multilayered media," Microwave Theory and Techniques, IEEE Transactions on, vol. 41, no. 1, pp. 79 -88, Jan. 1993.

[9] F. Olyslager and D. De Zutter, "Rigorous boundary integral equation solution for general isotropic and uniaxial anisotropic dielectric waveguides in multilayered media including losses, gain and leakage," Microwave Theory and Techniques, IEEE Transactions on, vol. 41, no. 8, pp. $1385-1392$, Aug. 1993.

[10] A. Yakovlev and G. Hanson, "Fundamental modal phenomena on isotropic and anisotropic planar slab dielectric waveguides," Antennas and Propagation, IEEE Transactions on, vol. 51, no. 4, pp. 888-897, 2003.

[11] — "Analysis of mode coupling on guided-wave structures using morse critical points," Microwave Theory and Techniques, IEEE Transactions on, vol. 46, no. 7, pp. 966-974, 1998.

[12] A. Poggio and E. Miller, "Integral equation solution of three-dimensional scattering problems," in Computer Techniques for Electromagnetics, R. Mittra, Ed. Pergamon Press, 1973, ch. 4.

[13] Y. Chang and R. Harrington, "A surface formulation for characteristic modes of material bodies," Antennas and Propagation, IEEE Transactions on, vol. 25, no. 6, pp. 789 - 795, Nov. 1977.

[14] T. Wu and L. Tsai, "Scattering from arbitrarily-shaped lossy dielectric bodies of revolution," Radio Science, pp. 709-718, Sep. 1977.

[15] J. Fostier, B. Michiels, I. Bogaert, and D. D. Zutter, "A Fast 2D Parallel MLFMA Solver for Oblique Plane Wave Incidence," Radio Science, Nov. 2011.

[16] F. Olyslager, Electromagnetic Waveguides and Transmission Lines. Oxford Engineering Science Series, 1999.

[17] T. Nishino, Function Theory in Several Complex Variables, ser. Translations of Mathematical Monographs. American Mathematical Society, 2001. 
[18] F. Olver, D. Lozier, R. Boisvert, and C. Clark, NIST Handbook of Mathematical Functions. Cambridge University Press, 2010.

[19] L. Hörmander, An introduction to complex analysis in several variables, ser. North-Holland mathematical library. North-Holland, 1990.

[20] R. Smith, S. N. Houde-Walter, and G. W. Forbes, "Mode determination for planar waveguide using the four-sheeted dispersion relation," Quantum Electronics, IEEE Journal of, vol. 28, no. 6, pp. 1520-1526, 1992.

[21] P. Henrici, Applied and Computational Complex Analysis, ser. Wiley Classics Library. Wiley, 1988.

[22] W. Press, S. Teukolsky, W. Vetterling, and B. Flannery, Numerical Recipes 3rd Edition: The Art of Scientific Computing, ser. Numerical Recipes: The Art of Scientific Computing. Cambridge University Press, 2007.

[23] J. Rhodes, "General constraints on propagation characteristics of electromagnetic waves in uniform inhomogeneous waveguides," Electrical Engineers, Proceedings of the Institution of, vol. 118, no. 7, pp. 849 -856 , Jul. 1971.

[24] R. Islam and G. Eleftheriades, "On the independence of the excitation of complex modes in isotropic structures," Antennas and Propagation, IEEE Transactions on, vol. 58, no. 5, pp. 1567-1578, 2010.

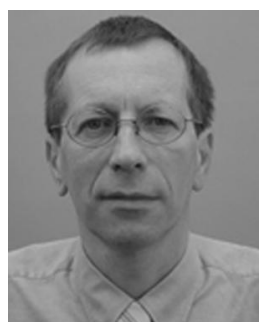

Daniël De Zutter was born in 1953. He received the M.Sc. degree in electrical engineering from Ghent University, Ghent, Belgium, in 1976. He received the Ph.D. degree in 1981, and he completed a thesis leading to a degree equivalent to the French Aggrégation or the German Habilitation in 1984.

He was a Research and Teaching Assistant at Ghent University from 1976 to 1984 . From 1984 to 1996, he was with the National Fund for Scientific Research, Flanders, Belgium. He is now Full Professor of electromagnetics, and the Head of the Department of Information Technology, Ghent University. He has served as the Dean of the Faculty of Engineering, Ghent University. Most of his earlier scientific work dealt with the electrodynamics of moving media. $\mathrm{He}$ has authored or co-authored more than 200 research papers published in international journals. His current research interests include all aspects of circuit and electromagnetic modeling of high-speed and high-frequency interconnections, packaging, on-chip interconnects, and numerical solutions of Maxwell's equations.

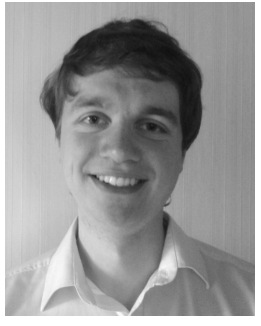

Dieter Dobbelaere was born in 1988. He received the M.Sc. degree in electrical engineering from Ghent University, Ghent, Belgium, in 2011. He is currently working toward the Ph.D. degree at the Electromagnetics Group of the Departement of Information Technology, Ghent University, as Research Fellow of the Fund for Scientific Research Flanders (FWO-V).

His research interests include full-wave analysis of electromagnetic waveguides, boundary integral formulations of Maxwell's equations and electro- magnetic wave theory.

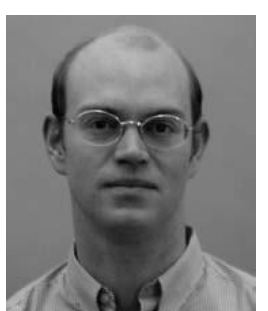

Hendrik Rogier Hendrik Rogier was born in 1971 He received the Electrical Engineering and the Ph.D. degrees from Ghent University, Gent, Belgium, in 1994 and in 1999, respectively. He is a currently a Full Professor with the Department of Information Technology of Ghent University, Belgium, Guest Professor at IMEC, Heverlee, Belgium, and Visiting Professor at the University of Buckingham, UK. From October 2003 to April 2004, he was a Visiting Scientist at the Mobile Communications Group of Vienna University of Technology. He authored and coauthored about 85 papers in international journals and about 100 contributions in conference proceedings. He is serving as a member of the Editorial Boarding of IET Science, Measurement Technology and acts as the URSI Commission B representative for Belgium. Within the IEEE Microwave Theory and Techniques Society, he is a member of Technical Committee 24 on RFID technology and within the European Microwave Association, he is a member of the Governing Board of Topical Group MAGEO on Microwaves in Agriculture, Environment and Earth Observation.

His current research interests are antenna systems, radiowave propagation, body-centric communication, numerical electromagnetics, electromagnetic compatibility and power/signal integrity.

Dr. Rogier was twice awarded the URSI Young Scientist Award, at the 2001 URSI Symposium on Electromagnetic Theory and at the 2002 URSI General Assembly. He is a Senior Member of the IEEE. 